\title{
Prevalence and Incidence of Microhemorrhages in Adolescent Football Players
}

\author{
(D)B.R. Shah, (D).M. Holcomb, (D)E.M. Davenport, (D) C.M. Lack, (D).M. McDaniel, (DD.M. Imphean, (D)Y. Xi, (DD.A. Rosenbaum,
} (D).E. Urban, (DB.C. Wagner, (D)A.K. Powers, (DC.T. Whitlow, (D).D. Stitzel, and (D).A. Maldjian

\begin{abstract}
BACKGROUND AND PURPOSE: SWI is an advanced imaging modality that is especially useful in cerebral microhemorrhage detection. Such microhemorrhages have been identified in adult contact sport athletes, and the sequelae of these focal bleeds are thought to contribute to neurodegeneration. The purpose of this study was to utilize SWI to determine whether the prevalence and incidence of microhemorrhages in adolescent football players are significantly greater than those of adolescent noncontact athletes.
\end{abstract}

MATERIALS AND METHODS: Preseason and postseason SWI was performed and evaluated on 78 adolescent football players. SWI was also performed on 27 adolescent athletes who reported no contact sport history. Two separate one-tailed Fisher exact tests were performed to determine whether the prevalence and incidence of microhemorrhages in adolescent football players are greater than those of noncontact athlete controls.

RESULTS: Microhemorrhages were observed in 12 football players. No microhemorrhages were observed in any controls. Adolescent football players demonstrated a significantly greater prevalence of microhemorrhages than adolescent noncontact controls $(P=.02)$. Although 2 football players developed new microhemorrhages during the season, microhemorrhage incidence during 1 football season was not statistically greater in the football population than in noncontact control athletes $(P=.55)$.

CONCLUSIONS: Adolescent football players have a greater prevalence of microhemorrhages compared with adolescent athletes who have never engaged in contact sports. While microhemorrhage incidence during 1 season is not significantly greater in adolescent football players compared to adolescent controls, there is a temporal association between playing football and the appearance of new microhemorrhages.

ABBREVIATION: SWI = susceptibility weighted imaging

M icrohemorrhages result in abnormal blood product and iron accumulation in the brain after vascular injury. Although microhemorrhages are commonly associated with hypertension, apolipoprotein E $\varepsilon 4$ carrier status, and cerebral

Received February 21, 2020; accepted after revision April 20.

From the Department of Radiology (B.R.S., J.M.H., E.M.D., J.M.M., D.M.I., Y.X., B.C.W., J.A.M.), University of Texas Southwestern Medical Center, Dallas, Texas; and Departments of Radiology (C.M.L., C.T.W.), Sports Medicine (D.A.R.), Biomedical Engineering (J.E.U., J.D.S.), and Neurosurgery (A.K.P.), Wake Forest School of Medicine, Winston-Salem, North Carolina.

Bhavya R. Shah and James M. Holcomb are co-first authors.

Support for this research was provided by National Institutes of Health grant R01NS082453 (J.A.M., J.D.S.) and R01NS091602 (C.T.W., J.A.M., J.D.S.).

Please address correspondence to James M. Holcomb, BSA, UT Southwestern, Department of Radiology, 5323 Harry Hines Blvd, Dallas, TX 75390-9178; e-mail: james.holcomb@utsouthwestern.edu

- Indicates open access to non-subscribers at www.ajnr.org

Indicates article with supplemental on-line table.

http://dx.doi.org/10.3174/ajnr.A6618 amyloid angiopathy, ${ }^{1}$ those found in healthy patients younger than 60 years of age are more likely the consequence of trauma. The deposition of such blood products in the brain has been implicated in a host of neurodegenerative disorders ${ }^{2-5}$ and has also been identified in athletes who participate in contact sports characterized by repetitive, high-magnitude head impacts. ${ }^{6-12}$ Collectively, these findings suggest that some neurodegenerative diseases, including chronic traumatic encephalopathy, may be the sequelae of transient, posttraumatic blood-brain barrier injury from sports-related head trauma. ${ }^{13}$

SWI is an advanced neuroimaging technique that is exquisitely sensitive to magnetic field disruption from the presence of highly paramagnetic and superparamagnetic blood products. Thus, SWI has markedly improved the sensitivity for detecting microhemorrhages. While microhemorrhage prevalences have been reported in adult contact sport cohorts, such rates in adolescent contact sport populations remain largely unexplored. ${ }^{14}$ The 
purpose of this study was to use SWI to determine whether the prevalence and incidence of microhemorrhages in adolescent football populations are significantly greater than those of adolescent, noncontact control athletes.

\section{MATERIALS AND METHODS \\ Participants}

All research procedures were approved by the institutional review board committees of Wake Forest School of Medicine and the University of Texas Southwestern School of Medicine. This study was compliant with the Health Insurance Portability and Accountability Act, and written informed parental consent and assent from the participants was obtained. Between 2012 and 2017, male adolescent football players were recruited via phone, e-mail, and in-person meetings from 6 Junior Pee Wee, 4 Pee Wee, 4 Junior Midget, one 10U, 1 Cadet, 2 Junior Varsity, and 4 Varsity football teams in Winston-Salem, North Carolina. Each football subject was enrolled in the study for the football season immediately following recruitment, with some subjects re-enrolling in the study in subsequent years. Male noncontact sport controls were recruited via phone, e-mail, and in-person meetings with noncontact sports leagues and extracurricular programs also located in Winston-Salem, North Carolina. Because hypertension is known to increase the risk of developing microhemorrhages, ${ }^{1}$ football and control participants were excluded from the analysis if they were hypertensive or taking medications known to elevate blood pressure. Medical histories revealed 1 football player using an angiotensin-converting-enzyme inhibitor and 14 football players and 7 control subjects using medications known to elevate blood pressure. To assess microhemorrhage incidence associated with playing football, football players were further excluded from analysis if preseason or postseason imaging was not acquired, imaging was limited by artifacts, or postseason imaging was not performed within 60 days from the conclusion of their season. Control subjects were excluded if medical histories revealed a prior concussion or head injury, imaging was limited by artifacts, or subjects had ever played, as defined by the American Academy of Pediatrics, a "collision" or "contact" sport. ${ }^{15}$ All control subjects were athletes who reported a sports history limited to baseball, swimming, tennis, and track.

\section{MR Imaging Acquisition}

MR imaging was performed on a 3T Skyra scanner (Siemens) with a 32-channel head coil. SWI was acquired under 2 protocols. The first SWI protocol parameters included the following: TR/ TE, 27/20 ms; flip angle, $15^{\circ}$; FOV, $232 \times 256$; section thickness, $1.5 \mathrm{~mm}$; and voxel resolution, $0.86 \times 0.86 \times 1.5 \mathrm{~mm}$. Fortyseven football subjects and 7 controls were scanned under the first protocol. The second SWI protocol parameters included the following: TR/TE, $51 / 9.75 \mathrm{~ms}$; flip angle, $20^{\circ}$; section thickness, $324 \times 416$; FOV, $2.0 \mathrm{~mm}$; and voxel resolution, $0.58 \times 0.58 \times$ $2.0 \mathrm{~mm}$. Thirty-one football subjects and 20 controls were scanned under the second protocol. T2 images were acquired using the following parameters: TR/TE, 3600/96 ms; flip angle, $160^{\circ}$; FOV , $640 \times 640$; section thickness, $3.0 \mathrm{~mm}$; and voxel resolution, $0.34 \times 0.34 \times 3.0 \mathrm{~mm}$. MR imaging data for all football participants were obtained before and after the football season. All MR imaging data for controls were obtained at 1 time point.

\section{SWI Analysis}

All scans were independently reviewed to identify hypointense foci on SWI by 2 board-certified, fellowship-trained neuroradiologists with $>10$ years of combined experience in practice. Each reviewer was blinded regarding subject cohort stratification. Following independent evaluation, a consensus conference was held to assess interrater agreement. Following consensus, 1 neuroradiologist examined corresponding phase and T2 images for each subject scan to exclude calcifications and cavernous malformations. Hypointensities on phase images were identified as calcifications. "Popcorn-shaped" lesions with a hemosiderin rim were identified as cavernous malformations on T2 imaging.

Prevalence in the football population was calculated as the total number of football players with postseason microhemorrhages divided by the total number of football players. Prevalence in the control population was calculated as the number of control subjects with microhemorrhages divided by the total number of controls.

To determine incidence in the football population, postseason microhemorrhages that were not identified on the corresponding subject's preseason image were classified as developed microhemorrhages. Incidence in the football population was calculated as the number of football players with developed microhemorrhages, divided by the total number of football players included in the analysis. Microhemorrhage prevalence in the control population was used as a proxy for microhemorrhage incidence in controls.

\section{Concussion Evaluation}

Concussion history before study enrollment was reported by the research subject and each player's legal guardians. During the season, the Sports Concussion Assessment Tool, 5th edition, was used to assist a certified athletic trainer in screening for concussion at all games and practices. ${ }^{16}$ If concussion was suspected, the subject was referred to a sports medicine physician to determine the diagnosis. As summarized by the American Medical Society for Sports Medicine Position Statement on concussion in sport, "Concussion remains a clinical diagnosis made by carefully synthesizing history and physical examination findings as the injury evolves. There is no specific imaging, biologic marker, or neuropsychological test currently able to confirm the diagnosis of sports concussion." 17

\section{Statistical Analysis}

The Cohen $\kappa$ coefficient was calculated to measure interrater agreement. A one-tailed Fisher test of exact probability was performed to determine whether microhemorrhage prevalence in adolescent football players was greater than that observed in the adolescent, noncontact controls. Similarly, a one-tailed Fisher test of exact probability was used to determine whether the incidence of microhemorrhages in adolescent football players was greater than that of adolescent noncontact controls. The $\alpha$ for all tests was set to .05 . To comply with the assumption of independent observations for each test and to avoid selection bias, 1 subject season was selected at random for the 18 football subjects with $>1$ usable season of data. Random selection was achieved using a random number generator. All statistical analyses and random number 

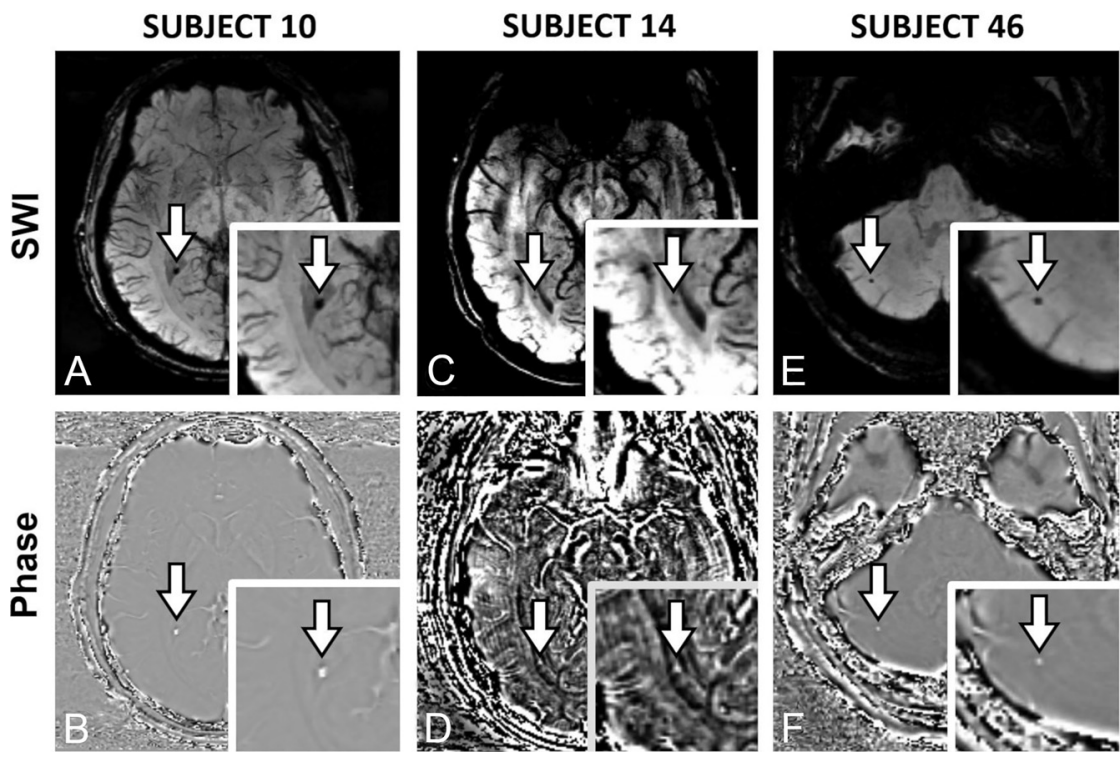

FIG 1. SWI and corresponding phase images. $A$ and $B$, Subject 10. $C$ and $D$, Subject 14. $E$ and $F$, Subject 46 . Subject 10 is an 18 -year-old, male defensive lineman. Subject 14 is a 17 -yearold, male defensive end. Subject 46 is an 18-year-old, male quarterback. Microhemorrhages are indicated by a white arrow. A, SWI hypointensity in the choroid plexus of the left lateral ventricle. $B$, Corresponding hyperintensity in the choroid plexus of the left lateral ventricle on phase imaging, verifying microhemorrhage. $C$, SWI hypointensity in the periventricular white matter of the left parietal lobe. $D$, Corresponding hyperintensity in the periventricular white matter of the left parietal lobe on phase imaging, verifying microhemorrhage. E, SWI hypointensity in the cerebellar gray/white matter. F, Corresponding hyperintensity in the cerebellar gray/white matter on phase imaging, verifying microhemorrhage.
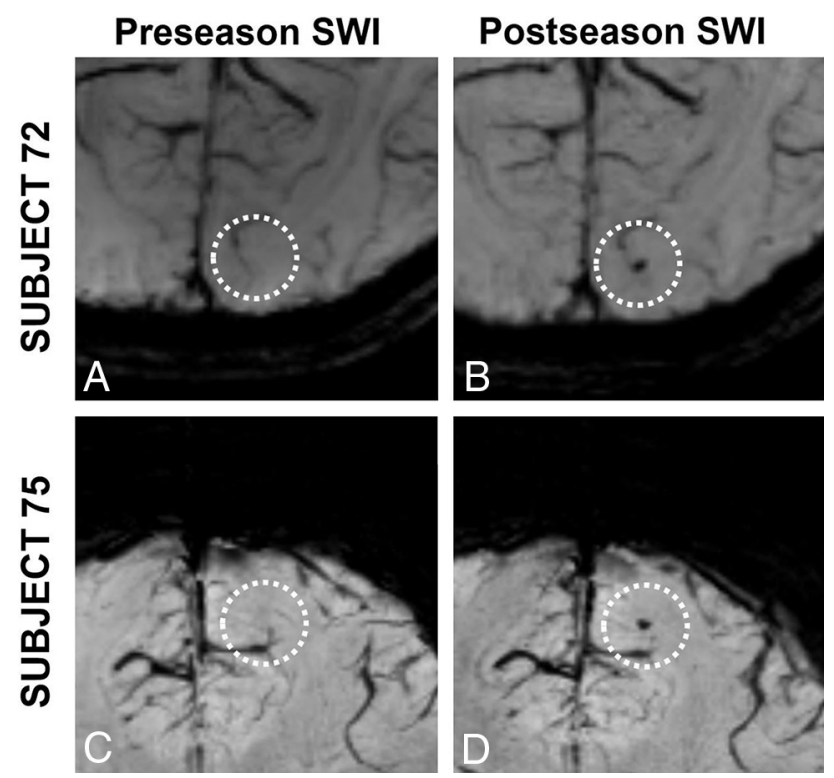

FIG 2. Developed microhemorrhages in 2 football players. Dashed circles identify the ROI on each image. $A$ and $B$, Subject 72. $C$ and $D$, Subject 75 . Subject 72 is a 17-year-old, male quarterback. Subject 75 is a 16-year-old, male cornerback. A, Preseason SWI with no hypointensity in the right occipital lobe white matter. B, Postseason SWI with hypointensity in the right occipital lobe white matter. C, Preseason SWI with no hypointensity in the right frontal lobe white matter. $D$, Preseason SWI with hypointensity in the right frontal lobe white matter. generation were performed in $\mathrm{R}$ 3.4.3 statistical and computing software (2017; http://www.r-project.org).

\section{RESULTS \\ Interrater Agreement}

The consensus conference revealed high interrater agreement in the determination of SWI lesion counts with $\kappa=1$.

\section{Population Demographics}

Following exclusion criteria and removal of duplicate subject seasons, 78 (39 pre-high school and 39 high school) male football players (age range, $10-18$ years; mean age, $14.25 \pm$ 2.63 years) and 27 adolescent noncontact controls (age range, 8-18 years; mean age $13.92 \pm 3.09$ years) were used for analysis.

\section{Adolescent Football Player \\ Summary}

Microhemorrhages were not detected in any control subjects. However, 12 football players had evidence of microhemorrhages on postseason imaging. Ten football players had the same microhemorrhages at baseline. Baseline microbleeds were identified in 2 offensive linemen, 2 defensive linemen, 2 defensive ends, 1 quarterback, 1 tight end, 1 fullback, and 1 wide receiver. These positions were self-reported and subject to change throughout a season, especially in pre-high school players. All pre-existing microhemorrhages were found in the lateral ventricle choroid plexus, white and gray matter of the cerebellum, periventricular white matter of the parietal lobe, and white matter of the brain stem, cerebellar peduncle, and frontal lobe (Fig 1). All football players with preseason microhemorrhages had played football for at least 1 year before study enrollment.

Two football players developed new microhemorrhages during the season. Subject 72, a quarterback, developed a microhemorrhage in the white matter of the occipital lobe, and subject 75, a cornerback, developed a microhemorrhage in the white matter of the frontal lobe (Fig 2). One pre-existing cavernous malformation (subject 33) was identified.

Of 12 football players who reported concussion history before study enrollment, 3 football players had baseline microhemorrhages. Neither of the 2 football players who developed new microhemorrhages reported a history of concussion nor were they diagnosed with a concussion during the football season. Nine football players were diagnosed with concussion during the season they were observed. None of the football players who were concussed during the season developed microhemorrhages or had microhemorrhages at baseline. A summary of data can be found in the On-line Table. 
Contingency table describing relationship between prior concussion diagnosis and microhemorrhage status in adolescent football players

\begin{tabular}{lccc}
\hline & Microhemorrhage Found & No Microhemorrhage Found & Total \\
\hline Prior concussion & 3 & 9 & 12 \\
No prior concussion & 9 & 57 & 66 \\
Total & 12 & 66 & 78 \\
\hline
\end{tabular}

professional fighters, collegiate hockey players, and former professional football players, respectively. ${ }^{6,8-10}$ Greater microhemorrhage prevalence in adolescent football players may reflect the unique intersection between sportsspecific biomechanics risks and a potentially greater vulnerability to

\section{Microhemorrhage Prevalence}

Of 78 football players, 13 microhemorrhages were identified in 12 subjects. Based on these observations, the microhemorrhage prevalence in adolescent football players is $15.38 \%$ (95\% confidence interval, $8.21 \%-25.33 \%$ ). No microhemorrhages were identified in the 27 controls. The microhemorrhage prevalence in the adolescent football population was significantly greater than that in the noncontact control population $(P$ value $=.02)$.

\section{Microhemorrhage Incidence}

Of 78 football players, 2 subjects developed new microhemorrhages during the season they were observed (subjects 72 and 75). Based on these results, the incidence of microhemorrhage among adolescent football players after one season is $2.56 \%(95 \%$ confidence interval, $0.31 \%-8.96 \%)$. None of the 27 noncontact controls developed microhemorrhages. This study did not demonstrate a greater incidence of microhemorrhage in adolescent football players compared with adolescent controls $(P$ value $=.55)$.

\section{Association Between Concussion and Microhemorrhage}

Using the Table, a post hoc $\chi^{2}$ test of independence was conducted to test the association between prior concussion diagnosis and microhemorrhage status. For analysis, subjects were considered concussed if they had self-reported a prior concussion diagnosis or were diagnosed with a concussion during the study. Postseason imaging was used to assess microhemorrhage status. No statistically significant relationship between prior concussion diagnosis and microhemorrhage status was observed $(P$ value $=.57)$.

\section{DISCUSSION}

This study demonstrates that there is a significantly greater prevalence of microhemorrhages in adolescent football players than in adolescent noncontact controls, with microhemorrhages preferentially occurring in older football players. Of the 13 total microhemorrhages, 11 were identified in high school football subjects and 2 were identified at the pre-high school level. This disparity could reflect a greater number of high-risk head impacts over a greater number of football seasons, or higher-magnitude head accelerations seen in older, more competitive levels of play. ${ }^{18,19}$ Despite a predilection for age, microhemorrhages did not preferentially occur on the basis of player position.

Microhemorrhage prevalence in adolescent contact sports has not been reported in the literature. However, several studies have documented such rates in adult contact sport athletes. The $15.38 \%$ microhemorrhage prevalence observed in our adolescent football population is greater than the prevalences of $9.52 \%$, $4.21 \%, 2.22 \%$, and $8.88 \%$, reported in amateur boxers, head injury among younger athletes. Indeed, boxers and professional football players, who demonstrate the highest microhemorrhage rates in the literature, experience similar inertial forces, peak translational head accelerations, and peak rotational head accelerations. $^{6,8-10,20}$ The acceleration risk of each head impact may be further magnified in adolescent athletes with underdeveloped neck strength and poor tackling technique. ${ }^{20-22}$

Although the prevalence of microhemorrhages is significantly greater in adolescent football players relative to noncontact controls, this study did not establish a statistically significant difference in incidence between groups. Across studies, microhemorrhages in contact sport athletes are a relatively rare finding with $90.48 \%-$ $97.78 \%$ of contact sport athletes demonstrating no evidence of microhemorrhages on SWI. ${ }^{6,8-10}$ Because microhemorrhage prevalence reflects the summation of incidences across broad time intervals, it is feasible that microhemorrhage incidence during 1 football season does not occur with enough frequency to yield statistically significant differences from controls. The abundance of baseline microhemorrhages relative to new microhemorrhages among our adolescent football players supports this notion and may further suggest that head impact intensity was greater in prior, unobserved seasons.

This study also did not establish a statistically significant relationship between concussion and the presence of microhemorrhages. Similarly, low microhemorrhage prevalence has been reported following pediatric concussion, ${ }^{23}$ in amateur boxers with no concussion history, ${ }^{6}$ and in professional fighters who experienced a high number of concussions and knockouts. ${ }^{9}$ Despite these findings, athletes have been known to mask concussion symptoms for a variety of reasons, ${ }^{24}$ and common concussion symptoms like headache and dizziness are not easily some concussions occurred in this study, but were not identified. Nevertheless, our results are consistent with other studies that report blood-brain barrier injury in contact sport athletes who exhibit no concussive symptoms. ${ }^{6,11,12}$

Although the sequelae of blood product-induced neurotoxicity and neurodegeneration are beyond the scope of this work, blood products alter normal choroid plexus function, neuronal electrical activity, and the composition of extracellular and cerebrospinal fluid. ${ }^{26-28}$ The presence of hemorrhagic products in the choroid plexus interstitium, paracellular spaces, and ventricles curtails choroid plexus cerebrospinal fluid production and impairs the ability of the choroid plexus to remove toxins and catabolites from the parenchymal extracellular environment. $^{26-28}$ After intraparenchymal hemorrhage, lysis of red blood cells starts at 24 hours and lasts several days. ${ }^{29}$ Red blood cell lysis releases hemoglobin, which is cytotoxic and results in brain edema, oxidative damage, and free radical injury.,30-32 observable, if not self-reported. ${ }^{25}$ As a result, it is possible that 
Hemoglobin is further broken down into heme and iron, which alters cell membrane permeability and electrical conduction and can ultimately result in cell death. ${ }^{5,30-32}$

There are several limitations of this study. Observations did not occur in a controlled environment where all nonfootball activities could be monitored. This limitation is especially true of subjects who had microhemorrhages at baseline. As a result, there is the possibility that microhemorrhages originated as a consequence of other contact sports or other non-sports-related activities. In the football players with baseline microhemorrhages and known sports history, 3 subjects exclusively played football, 1 subject previously played lacrosse and basketball, and another subject previously played lacrosse and soccer. While a relationship may exist between microhemorrhage development and other contact sports, all subjects with microhemorrhages had a history of playing football. Moreover, the 2 football players who developed microhemorrhages during the football season provide evidence of at least a temporal relationship between microhemorrhage development and football participation.

This study is further limited by controversy within the literature on the extent of microhemorrhage resorption. In a study of 13 combat veterans, it was found that $34 \%$ of all microhemorrhages disappeared completely from SWI 1 year after the initial scan, ${ }^{33}$ while a longitudinal study of 26 civilians demonstrated that all SWI microhemorrhages at baseline were still present 8 years later, albeit with reduced volume. ${ }^{34}$ If SWI microhemorrhages become undetectable with time, the prevalence and incidence of microhemorrhages in both football and control populations may be underreported. The imaging modalities also have some limitations. Due to susceptibility artifacts, SWI performs poorly in revealing lesions near bone-air interfaces. Additionally, neither SWI nor T2 imaging can easily distinguish microhemorrhages from small cavernous malformations. Both imaging limitations may have respectively deflated and inflated the number of identified microhemorrhages.

\section{CONCLUSIONS}

This study demonstrates that the prevalence of microhemorrhages in adolescent football players is significantly greater than that observed in adolescent noncontact control athletes. Although microhemorrhage incidence is not significantly greater in adolescent football players compared with adolescent controls, there is a temporal association between playing football and the appearance of new microhemorrhages. Furthermore, this study does not demonstrate a statistically significant relationship between concussion diagnosis and microhemorrhage prevalence in adolescent football players.

Disclosures: Elizabeth M. Davenport-RELATED: Grant: National Institutes of Health, Comments: R01*; UNRELATED: Grants/Grants Pending: National Institutes of Health, Comments: R01.* Christopher M. Lack—UNRELATED: Employment: Wake Forest School of Medicine, Comments: employed as a radiologist; Expert Testimony: law firms, Comments: expert witness in neuroradiology; none related to football injuries. Daryl A. Rosenbaum-RELATED: Grant: National Institutes of Health, Comments: iTAKL study grant.* Jillian E. UrbanRELATED: Grant: National Institutes of Health, Comments: R01NS082453, R01NS091602, R01NS094410*; UNRELATED: Grants/Grants Pending: R01NS119907, R01NS118243, R01NS119766-01 (as co-investigator) and K25HD101686 pending. The $\mathrm{K} 25$ is directly related to youth football and proposes to develop and test head impact intervention in the sport.* Patents (Planned, Pending or Issued): We have a patent pending for an instrumented mouthpiece form factor. No money has been paid to us or the institution. Benjamin C. Wagner-RELATED: Grant: National Institutes of Health, Comments: National Institutes of Health grant R01NS082453 and R01NS091602. Christopher T. Whitlow-RELATED: Grant: National Institutes of Health, Comments: R01NS091602.* Joel D. StitzelRELATED: Grant: National Institutes of Health, Comments: R01NS082453, R01NS091602.* Joseph A. Maldjian-RELATED: Grant: National Institutes of Health, Comments: R01NS082453, R01NS091602.* *Money paid to the institution.

\section{REFERENCES}

1. Poels MM, Vernooij MW, Ikram MA, et al. Prevalence and risk factors of cerebral microbleeds: an update of the Rotterdam scan study. Stroke 2010;41:S103-06 CrossRef Medline

2. Nisenbaum EJ, Novikov DS, Lui YW. The presence and role of iron in mild traumatic brain injury: an imaging perspective. J Neurotrauma 2014;31:301-07 CrossRef Medline

3. Li K, Reichmann H. Role of iron in neurodegenerative diseases. $J$ Neural Transm (Vienna) 2016;123:389-99 CrossRef Medline

4. Cordonnier C, van der Flier WM. Brain microbleeds and Alzheimer's disease: innocent observation or key player? Brain 2011;134:335-44 CrossRef Medline

5. Núñez MT, Urrutia P, Mena N, et al. Iron toxicity in neurodegeneration. Biometals 2012;25:761-76 CrossRef Medline

6. Hasiloglu Z, Albayram S, Selcuk H, et al. Cerebral microhemorrhages detected by susceptibility-weighted imaging in amateur boxers. AJNR Am J Neuroradiol 2011;32:99-102 CrossRef Medline

7. Casson IR, Viano DC, Haacke EM, et al. Is there chronic brain damage in retired NFL players? Neuroradiology, neuropsychology, and neurology examinations of 45 retired players. Sports Health 2014; 6:384-95 CrossRef Medline

8. Jarrett M, Tam R, Hernández-Torres E, et al. A prospective pilot investigation of brain volume, white matter hyperintensities, and hemorrhagic lesions after mild traumatic brain injury. Front Neurol 2016;7:11 CrossRef Medline

9. Lee J, Wu J, Banks S, et al. Prevalence of traumatic findings on routine MRI in a large cohort of professional fighters. AJNR Am J Neuroradiol 2017;38:1303-10 CrossRef Medline

10. Kuhn AW, Zuckerman SL, Solomon GS, et al. Interrelationships among neuroimaging biomarkers, neuropsychological test data, and symptom reporting in a cohort of retired National Football League players. Sports Health 2017;9:30-40 CrossRef Medline

11. Slobounov SM, Walter A, Breiter HC, et al. The effect of repetitive subconcussive collisions on brain integrity in collegiate football players over a single football season: a multi-modal neuroimaging study. Neuroimage Clin 2017;14:708-18 CrossRef Medline

12. Hähnel S, Stippich C, Weber I, et al. Prevalence of cerebral microhemorrhages in amateur boxers as detected by 3T MR imaging. AJNR Am J Neuroradiol 2008;29:388-91 CrossRef Medline

13. Tagge CA, Fisher AM, Minaeva OV, et al. Concussion, microvascular injury, and early tauopathy in young athletes after impact head injury and an impact concussion mouse model. Brain 2018;141:42258 CrossRef Medline

14. Kulich M, Fisher LM, Voelker C. Imaging findings in mild traumatic brain injury. In: Hoffer M, Balaban D, eds. Neurosensory Disorders in Mild Traumatic Brain Injury. Academic Press; 2018: 23-47

15. Committee on Sports Medicine and Fitness. Medical conditions affecting sports participation. Pediatrics 1994;94:757-60

16. Echemendia RJ, Meeuwisse W, McCrory P, et al. The sport concussion assessment tool 5 th edition (SCAT5): background and rationale. Br J Sports Med 2017;51:848-50 CrossRef Medline

17. Harmon KG, Drezner J, Gammons M, et al; American Medical Society for Sports Medicine. American Medical Society for Sports Medicine Position Statement: concussion in sport. Clin J Sport Med 2013;23:1-18 CrossRef Medline

18. Kelley ME, Urban JE, Miller LE, et al. Head impact exposure in youth football: comparing age-and weight-based levels of play. $J$ Neurotrauma 2017;34:1939-47 CrossRef Medline 
19. Urban JE, Davenport EM, Golman AJ, et al. Head impact exposure in youth football: high school ages 14 to 18 years and cumulative impact analysis. Ann Biomed Eng 2013;41:2474-87 CrossRef Medline

20. Viano DC, Casson IR, v EJ, et al. Concussion in professional football: comparison with boxing head impacts, Part 10. Neurosurgery 2005;57:1154-72 CrossRef Medline

21. Broglio SP, Sosnoff JJ, Shin S, et al. Head impacts during high school football: a biomechanical assessment. J Athl Train 2009;44:342-49 CrossRef Medline

22. Collins CL, Fletcher EN, Fields SK, et al. Neck strength: a protective factor reducing risk for concussion in high school sports. J Prim Prev 2014;35:309-19 CrossRef Medline

23. Bonow RH, Friedman SD, Perez FA, et al. Prevalence of abnormal magnetic resonance imaging findings in children with persistent symptoms after pediatric sports-related concussion. J Neurotrauma 2017;34:2706-12 CrossRef Medline

24. Kroshus E, Garnett B, Hawrilenko M, et al. Concussion underreporting and pressure from coaches, teammates, fans, and parents. Soc Sci Med 2015;134:66-75 CrossRef Medline

25. Echlin PS, Tator $\mathrm{CH}$, Cusimano MD, et al. Return to play after an initial or recurrent concussion in a prospective study of physicianobserved junior ice hockey concussions: implications for return to play after a concussion. Neurosurg Focus 2010;29:E5 CrossRef Medline

26. Johanson C, Stopa E, Baird A, et al. Traumatic brain injury and recovery mechanisms: peptide modulation of periventricular neurogenic regions by the choroid plexus-CSF nexus. J Neural Transm (Vienna) 2011;118:115-33 CrossRef Medline

27. Lindvall M, Edvinsson L, Owman C. Sympathetic nervous control of cerebrospinal fluid production from the choroid plexus. Science 1978;201:176-78 CrossRef Medline

28. Spector R, Johanson CE. Choroid plexus failure in the Kearns-Sayre syndrome. Cerebrospinal Fluid Res 2010;7:14 CrossRef Medline

29. Wagner KR, Sharp FR, Ardizzone TD, et al. Heme and iron metabolism: role in cerebral hemorrhage. J Cereb Blood Flow Metab 2003;23:629-52 CrossRef Medline

30. Aronowski J, Zhao X. Molecular pathophysiology of cerebral hemorrhage: secondary brain injury. Stroke 2011;42:1781-86 CrossRef Medline

31. Huang FP, Xi G, Keep RF, et al. Brain edema after experimental intracerebral hemorrhage: role of hemoglobin degradation products. J Neurosurg 2002;96:287-93 CrossRef Medline

32. Zheng $\mathrm{H}$, Chen $\mathrm{C}$, Zhang J, et al. Mechanism and therapy of brain edema after intracerebral hemorrhage. Cerebrovasc Dis 2016;42:15569 CrossRef Medline

33. Liu W, Soderlund K, Senseney JS, et al. Imaging cerebral microhemorrhages in military service members with chronic traumatic brain injury. Radiology 2016;278:536-45 CrossRef Medline

34. Daugherty AM, Raz N. Incident risk and progression of cerebral microbleeds in healthy adults: a multi-occasion longitudinal study. Neurobiol Aging 2017;59:22-29 CrossRef Medline 